Gut, 1989, 30, 1329-1333

\title{
Profound spatial clustering of simultaneous peptic ulcers
}

\author{
MITCHELL S CAPPELL \\ From the Division of Gastroenterology of the Department of Medicine, University of Medicine of New Jersey, \\ Robert Wood Johnson (Rutgers) Medical School, New Brunswick, New Jersey, USA
}

SUMmaRy In an endoscopic study of 90 consecutive patients with more than one peptic ulcer, the ulcers in an individual were profoundly spatially clustered. Clustering of ulcer locations was shown using a non-parametric test of clustering (Kruskal-Wallis statistic with 89 degrees of freedom= $151 \cdot 31$, probability of observing this extreme statistic with no clustering $<0 \cdot 0005$ ) and a parametric test of clustering ( $F$ test statistic with 89 and 124 degrees of freedom $=5 \cdot 41$, probability of observing this extreme statistic with no clustering $<0 \cdot 0005)$. Patients having their largest ulcer in any given region had a much greater likelihood than other patients of having other ulcers in that same site. For example, the 26 patients with their largest ulcer in the proximal duodenal bulb had 20 of 33, or $61 \%$ (9) (standard error), of their other ulcers in the proximal duodenal bulb. In contrast, the 18 patients with their largest ulcer in the proximal stomach had four of 23 , or $17 \%(8)$, of their other ulcers in the proximal duodenal bulb. Of the 59 patients who had two simultaneous ulcers, 28 patients had adjacent ulcers (distance between ulcers less than $4 \%$ of the distance from the gastric cardia to the apex of the duodenal bulb). These findings suggest that local factors may be important in the pathogenesis of simultaneous peptic ulcers, including infection caused by Campylobacter pylori or other microorganisms, ischaemia and mucosal barrier disruption.

Notwithstanding the apparent heterogeneity of peptic ulcer disease,,$^{1-5}$ an important question is the overall role of non-local factors relative to local gastroduodenal factors in the pathogenesis of peptic ulcers. Proposed non-local systemic, general gastroduodenal and environmental factors in peptic ulcer pathogenesis include abnormalities in serum hormone levels, ${ }^{, 7}$ immunity, ${ }^{, "}$ intraluminal $\mathrm{pH}, 21111$ intraluminal enzyme concentrations, ${ }^{12}$ and environmental stress. This work shows remarkably profound spatial clustering of simultaneous peptic ulcers in individuals. This finding suggests that local factors are important in the pathogenesis of simultaneous peptic ulcers.

\section{Methods}

PATIENTS

From 1984 through 1987, 90 patients without a

Address for correspondence: Dr Mitchell S Cappell. Department of Medicine/ (iastroenterology. University of Medicine of New Jersey. Robert Wood Johnson Medical School, New Brunswick, New Jersey (1890)3-(1019, USA.

Accepted for publication 14 Fehruary 1989. history of previous gastric or duodenal surgery had multiple peptic ulcers found in the stomach and duodenal bulb by oesophagogastroduodenoscopy at the Bronx Municipal Hospital Center of the Albert Einstein College of Medicine. Ulcer locations, determined at oesophagogastroduodenoscopy, were recorded on a diagram of the stomach and duodenum. These data were digitized using a Hewlett Packard 9864A digitizer with a scale of 1 for the proximal gastric cardia up to 50 for the distal apex of the duodenal bulb. The distance between two ulcers equalled the difference between their assigned numbers. Gastric ulcers not near or in the pylorus were standardly biopsied and followed until healing to exclude malignancy. All biopsy specimens were reviewed by the investigator. Pathological examination of these biopsied ulcer margins confirmed in $47 \%$ of cases and supported (finding of acute and chronic inflammation) in $53 \%$ of cases the endoscopic diagnosis of a peptic ulcer. This study excluded nonpeptic ulcers caused by adenocarcinoma, lymphoma or other neoplasms, ulcers associated with Mycohacterium avium intracellulare in one patient and with Herpes simplex in one patient, ulcers caused by 
an indwelling nasogastric tube in two patients, and superficial (stress) ulcers in five patients. Biopsy specimens were not routinely tested for Campylobacter pylori (Campylobacter pyloridis) and peptic ulcers associated with Campylobacter pylori were not excluded. None of the patients had a gastrinoma. The primary indication for oesophagogastroduodenoscopy was: gastrointestinal bleeding in 61 , abdominal pain in 14 , an abnormal upper gastrointestinal series in 10 and other in five. Eleven patients had been taking ulcerogenic medications, including acetylsalicylic acid, indomethacin and ibuprofen, before the diagnosis of multiple ulcers; this group was too small to statistically analyse separately.

STATISTICAL ANALYSIS

Samples of a clustered population may exhibit greater clustering. ${ }^{13}$ For example, the location distribution of people in the United States is highly clustered (in cities). But the location distribution of people in a family is even more clustered because all family members usually live in the same house. The location of ulcers in the population is somewhat clustered in the duodenal bulb, pyloric channel and gastric incisura. Ulcer locations in individuals were shown to have greater clustering, however - that is, ulcers closer in an individual than expected by their location distribution in the population.

First, the ulcer locations in the 90 individuals were compared using the Kurskal-Wallis test, a nonparametric one-way analysis of variance on the ordered sites with 90 samples. For this test ulcer locations were divided into five ordered regions: proximal stomach (location 1-25), antrum (26-35), pyloric region (36-40), proximal duodenal bulb (41-45) and distal duodenal bulb (46-50). For each patient the number of ulcers at each site were calculated. The Kruskal-Wallis statistic (method 1$)$ with 89 degrees of freedom, then tests the null hypothesis that the distribution of ulcers across sites is the same for all patients.

Second, clustering of ulcer locations in individuals was tested using a parametric one-way analysis of variance with an $\mathrm{F}$ test (performed on the 90 patients and 214 ulcers) with 89 and 124 degrees of freedom (method 2). The observations on each patient were the measured location of each ulcer on a scale of 1 to 50. The F statistic tests the null hypothesis that the distribution of measurements is the same for each patient.

\section{Results}

NON-PARAMETRIC TEST OF CLUSTERING

The Kruskal-Wallis method tests the null hypothesis that the probability distributions of ulcer locations in the 90 patients were identical. ${ }^{1+}$ With 89 degrees of freedom, the Kruskal-Wallis statistic for the 90 patients was 151.31 (method 1). Thus the null hypothesis of no clustering was rejected at a 0.0005 level of confidence (critical value of cumulative $\chi^{2}$ with 89 degrees of freedom $=139 \cdot 5$ ).

PARAMETRIC TEST OF CLUSTERING

The null hypothesis of a homogeneous location distribution in the 90 patients was rejected using an $\mathrm{F}$ test (method 2 ) with 89 and 124 degrees of freedom at a 0.0005 level of confidence $(\mathrm{F}=5 \cdot 41>1 \cdot 88=$ $\left.\mathrm{F}(0 \cdot 0005)_{89.124}\right)$.

OTHER ULCERS NEAR LARGEST ULCER

If ulcers occur independently within the same patient, then the distribution of smaller, secondary ulcers should be independent of the site of the primary largest ulcer. ${ }^{13}$ Figures $1 \mathrm{~b}$ to $1 \mathrm{e}$ show that this did not occur, however. The patients had a greater likelihood of having their other ulcers in the same region as their largest ulcer. For example, the 26 patients with their largest ulcer in the proximal duodenal bulb had $61 \%$ (9) (or 20 out of 33) of their secondary ulcers in the proximal duodenal bulb (Fig. 1d). In contrast, the 18 patients with their largest ulcer in the proximal stomach had $17 \%$ (18) (or four of 23 ) of their secondary ulcers in the proximal duodenal bulb (Fig. 1b). Also, patients had a smaller likelihood of having their other ulcers in regions remote from their largest ulcer (Fig. 1b to 1e).

Other ulcers were as close to a patient's smallest as compared with a patient's largest ulcer. For example, patients with their smallest or with their largest ulcer in the distal duodenal bulb had similar distributions of their other ulcers (not illustrated, $\mathrm{p} \gg 0 \cdot 10$, $\chi^{2}=1 \cdot 48$, degrees of freedom $=4$ ).

\section{FREQUENTLY ADJACENT UICERS}

Of 59 patients with two simultaneous ulcers, 28 patients had adjacent ulcers (distance between ulcers in the longitudinal direction less than or equal to $4 \%$ of the distance from the gastric cardia to the apex of the duodenal bulb).

\section{Discussion}

This report extends previously reported findings. Gastric ulcers associated with duodenal ulcer or deformity tended to be more frequently prepyloric and antral. ${ }^{15-19}$ Patients with a gastric ulcer were more likely to have other gastric than duodenal ulcers." The reported tendency to develop new ulcers near a prior ulcer site"l may be because of spatial clustering of new ulcers near a previous ulcer or merely because 


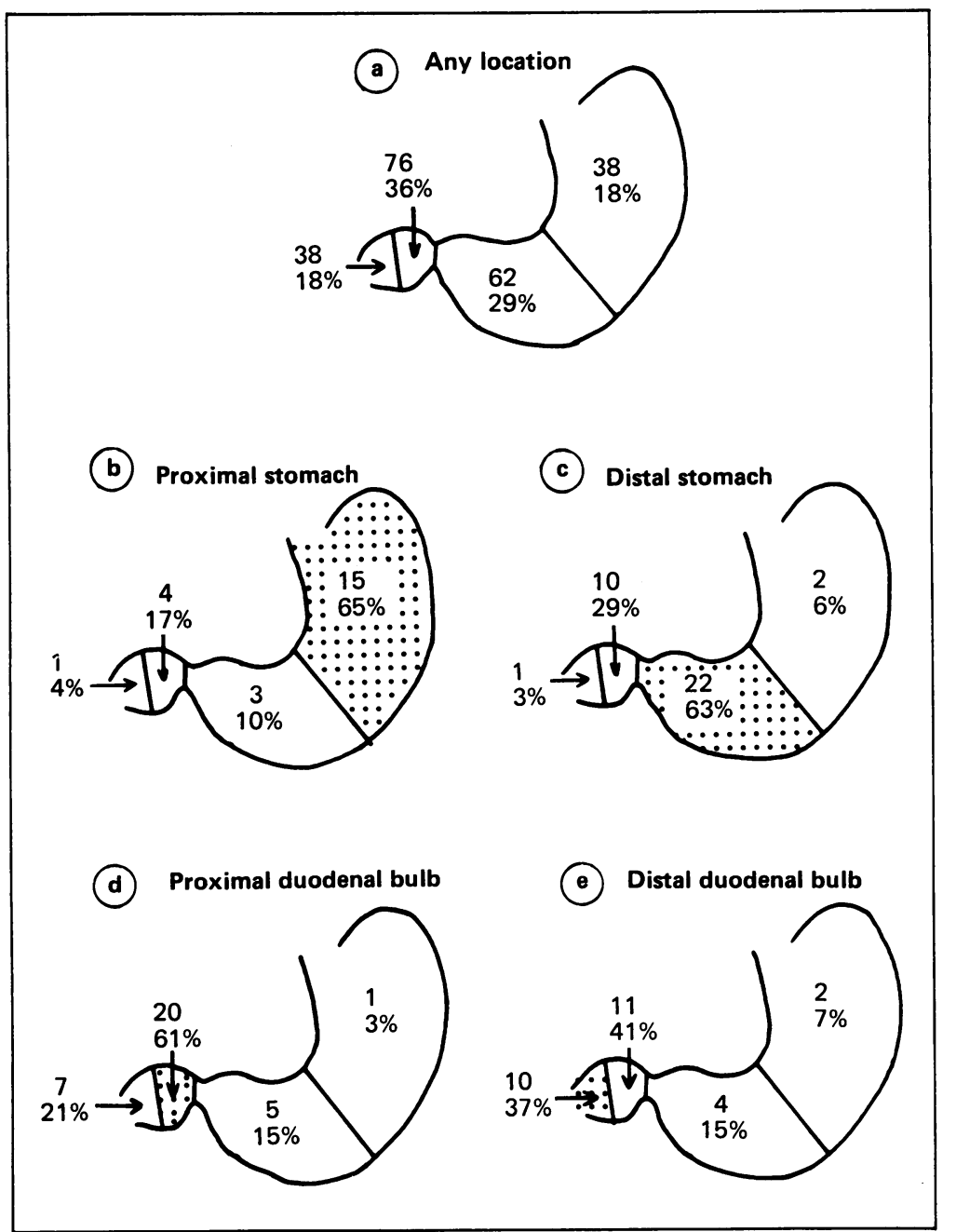

Fig. 1 The stomach and duodenum were divided into four regions. (a) all ulcers in all 90 patients, b-c patients'secondary, smaller ulcers in patients with their largest ulcer located in the: proximal stomach (b), distal stomach including the antrum and pyloric region (c), proximal duodenal bulb (d) and distal duodenal bulb $(e)$. The frequency of secondary ulcers being in the same (stippled) region as the patient's largest ulcer in (b-c) was markedly greater than expected without clustcring. For example, in b the 18 patients with their largest ulcer in the proximal stomach had $65 \%$ (10) (standard error) or 15 of 23 of their secondary ulcers in the same region. In contrast, in e the 19 patients with their largest ulcer in the distal duodenum had $7 \%$ (5) or two of 27 of their secondary ulcers in the proximal stomach. Also, the frequency of simultaneous ulcers being in a region remote from the largest ulcer in b-c was less than expected without clustering. Numbers of ulcers were 214, 23, 35, 33 and 27 in a to c, respectively. Numbers of patients were 90,18,27, 26 and 19 in a through c, respectively.

of ulcer recurrence. First degree relatives of patients with gastric ulcers had an increase in the prevalence of gastric ulcer, but not of duodenal ulcer. Relatives of patients with duodenal ulcers had an increase in the prevalence of duodenal ulcer, but not of gastric ulcer. ${ }^{12-2-24}$ The clustering of multiple ulcers at the same locus in individuals and their relatives can be explained by a genetic predisposition to ulceration at a specific site.

Spatial clustering shows the importance of local factors, possibly including local mucosal histology, ischaemia, ${ }^{25}$ in infection with Campylobacter pyloris ${ }^{27-}$ ${ }_{2 y}$ or other microorganisms, or defective cytoprotection ${ }^{17.34: 32}$ in the pathogenesis of multiple peptic ulcers. 
Local ischaemia produces skin ulcers clustered near the medial maleolus in patients with sickle cell disease." Analysis of differences between mucosa near to and remote from ulcers may elucidate the mechanism of ulcer clustering.

Patients with multiple ulcers represented $20.5 \%$ of all patients with peptic ulcers found on oesophagogastro-duodenoscopy during the present study. Some evidence supports that patients with multiple duodenal ulcers ${ }^{i+}$ and concomitant gastric and duodenal ulcers ${ }^{23}$ in have a different disease than patients with single ulcers. Some evidence supports that patients with multiple gastric ulcers is or concomitant gastric and duodenal ulcers ${ }^{11}$ have a similar disease as patients with single ulcers.

I would like to thank Mrs Sharon Hunt for typing this manuscript.

\section{References}

1 Ellis A. The genetics of peptic ulcer. Scand J Gastroenterol 1985: 110: 25-7.

2 Johnson HD. Gastric ulcer: classification. blood group characteristics, secretion patterns and pathogenesis. Ann Surg 1965: 162: 996-10(04.

3 Kirk RM. Are gastric and duodenal ulcers separate discases or do they form a continuum? Dig Dis Sci 1981: 26: $149-54$

4 Rotter JI. Gastric and duodenal ulcer are each many different diseases. Dig Dis Sci 1981; 26: 1.54-60).

5 Vesely KT, Kubickova Z. Dvorakova M. Zvolankova $K$. Clinical data and characteristics differentiating types of peptic ulcer. Gut 1968: 9: 57-68.

6 Isenberg JI. Cano R. Bloom SR. Effect of graded amounts of acid instilled into the duodenum on pancreatic bicarbonate secretion and plasma secretin in duodenal ulcer patients and normal subjects. Gastroenterology 1977: 72: 6-8.

7 McLoughlin JC, Green WER. Buchanan KD. Gastric emptying of ingested acid and its effects on plasma gastrin and secretin in duodenal ulcer subjects. Scand $J$ Gastroenterol 1978; 13: 313-9.

8 Baenkler HW. Domschke W. Domschke S. Wunsch E. Jacger E. Demling L. Duodenal ulcer - an immunological discase? Acta Hepato-Gastroenterol 1975; 22: 209-10.

9 Rotter JI. Heiner DC. Are there immunologic forms of duodenal ulcer? J Clin Lab Immunol 1982: 7: 1-6.

10 Bodemar G. Walon A. Lundquist G. Food stimulated acid secretion measured by intragastric titration with bicarbonate in patients with duodenal and gastric ulcer discase and in controls. Scand J Gastroenterol 1978; 13: 911-8.

11 Malagelada JR. Longstreth GF. Deering TB. Summerskill WHJ, Go VLW. Gastric secretion and emptying after ordinary meals in duodenal ulcer. Gastroenterology 1977: 73: 989-94.

12 Rotter JI, Sones JQ. Samloff IM. et al. Duodenal ulcer disease associated with elevated serum pepsinogen I: an inherited autosomal dominant disorder. $N$ Engl $J$. Med 1979: 300: 63-6.

13 Cappell MS. Forde KA. Spatial clustering of multiple hyperplastic. adenomatous and malignant colonic polyps in individual patients. Dis Colon Rectum 1989: 32: $641-52$.

14 Ostle B. Malone LC. Statistics in research: Basic concepts and techniques for research workers. Fourth edition. Ames. Iowa. Iowa University Press. 1988: 176-203.

1.5 Blackstone MO. Benign gastric ulcers. In: Endoscopic interpretation: normal and pathologic appearances of the gastrointestinal tract. New York: Raven Press, 1984: 121-36.

16 Bonnevie O. Gastric and duodenal ulcers in the same patient. Scand J Gastroenterol 1975: 10: 657-774.

17 Oi M. Oshida K. Sugimura S. The location of gastric ulcer. Gastroenterology 1959: 36: 4.5-6.

18 Roth JLA. Diagnosis of peptic ulcer: clinical aspects. In: Berk JE. Haubrich WS. Kalsen MH. Roth JLA. Schaffner F. eds. Bockus Gastroenterology. th edition. Philadelphia. Saunders. 1985: 1060-88.

19 Rumball JM. Coexistent duodenal ulcer. Gastroenterology 1971:61: 622-7.

20) Dolphin JA. Smith LA. Waugh JM. Multiple gastric ulcers: their occurrence in benign and malignant lesions. Gastroenterology 1953:25: 202-5.

21 Littman A. Hanscom DH. The course of recurrent ulcer. Gastroenterology 1971: 61: 592-7.

22 Doll R. Kellock TD. The separate inheritance of gastric and duodenal ulcers. Ann Eugenics 1951; 16: 231-40.

23 Eberhard G. Peptic ulcer in twins: a study in personality. heredity, and environment. Acta Psychiatry Scand 1968: 205 [suppl]: 1-118.

24 Tarpila S. Samloff IM. Pikkarainen P. Vuoristo M. Ihamaki T. Endoscopic and clinical findings in firstdegree relatives of duodenal ulcer patients and control subjects. Scand J Gastroenterol 1982; 17: 50)3-6.

25 Pihan G. Majzoubi D. Haudenschild C. Trier JS. Szabo S. Early microcirculatory stasis in acute gastric mucosal injury in the rat and prevention by 16, 16-dimethyl prostaglandin $\mathrm{E}_{2}$ or sodium thiosulfate. Gastroenterology 1986; 91: 1415-26.

26 Szabo S. Trier JS, Brown A. Schnoor J. Early vascular injury and increased vascular permeability in gastric mucosal injury caused by ethanol in the rat. Gastroenterology 1985: 88: 228-36.

27 Blaser MJ. Gastric Campylobacter-like organisms. gastritis, and peptic ulcer discase. Gastroenterology 1987: 93: 371-83.

28 Dooley CP. Cohen $\mathrm{H}$. The clinical significance of Campylobacter pylori. Ann Intern Med 1988: 108: 70-9.

29 Price AB. Levi J. Dolby JM. et al. Campylobacter pyloridis in peptic ulcer disease: microbiology, pathology, and scanning electron microscopy. Gut 1985; 26: $1183-8$

30) Oi M. Ito Y. Kumagai F. et al. A possible dual control mechanism in the origin of peptic ulcer. Gastroenterology 1969: 57: 280-93.

31 Turnberg LA. Gastric mucosal defense mechanism. Scand J Gastroenterol 1985: 20 [suppl 110): 37-40.

32 Youran F. Pearson J. Allen A. Venables C. Changes in 
the structure of the mucosal surface of the stomach in association with peptic ulcer disease. Gastroenterology 1982; 82: 827-31.

33 Serjeant GR. Leg ulceration in sickle cell ancmia. Arch Intern Med 1974; 133: 690-4.

34 Hui WH, Lam SK. Multiple duodenal ulcer: natural history and pathophysiology. Gut 1987; 28: 1134-41.

35 Lam SK. Lai CL. Gastric ulcers with and without associated duodenal ulcer have different pathophysiology. Clin Sci Mol Med 1978; 55: 97-102.
36 McCray RS, Ferris EJ, Herskovic T, Winawer SJ, Shapiro JH. Zamcheck N. Clinical differences between gastric ulcers with and without duodenal deformity. Ann Surg 1968; 168: 821-823.

37 Boyle JD. Multiple gastric ulcers. Gastroenterology 1971: 61: 628-40.

38 Degradi AE. Falkner RE. Lec ER. Multiple benign gastric ulcers: a clinical and gastroscopic study. $A m \mathrm{~J}$ Gastroenterol 1974: 62: 36-45. 